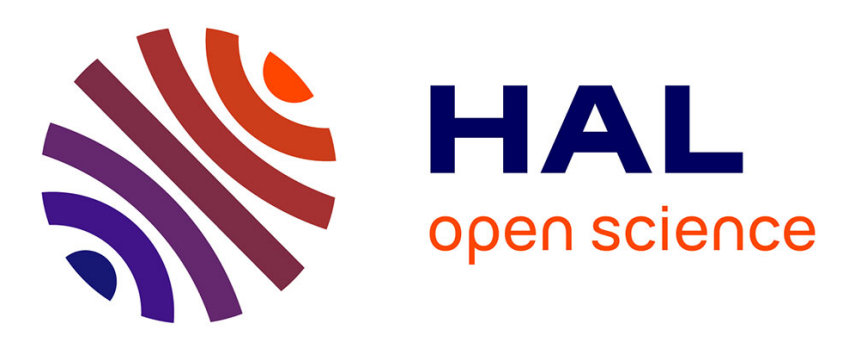

\title{
Modeling Novel Soft Mechanosensors based on Air-Flow Measurements
}

\author{
Stefan Escaida Navarro, Olivier Goury, Gang Zheng, Thor Morales Bieze, \\ Christian Duriez
}

\section{- To cite this version:}

Stefan Escaida Navarro, Olivier Goury, Gang Zheng, Thor Morales Bieze, Christian Duriez. Modeling Novel Soft Mechanosensors based on Air-Flow Measurements. IEEE Robotics and Automation Letters, 2019, 4 (4), pp.4338 - 4345. 10.1109/LRA.2019.2932604 . hal-02239080

\section{HAL Id: hal-02239080 \\ https://hal.inria.fr/hal-02239080}

Submitted on 1 Aug 2019

HAL is a multi-disciplinary open access archive for the deposit and dissemination of scientific research documents, whether they are published or not. The documents may come from teaching and research institutions in France or abroad, or from public or private research centers.
L'archive ouverte pluridisciplinaire HAL, est destinée au dépôt et à la diffusion de documents scientifiques de niveau recherche, publiés ou non, émanant des établissements d'enseignement et de recherche français ou étrangers, des laboratoires publics ou privés. 


\title{
Modeling Novel Soft Mechanosensors based on Air-Flow Measurements
}

\author{
Stefan Escaida Navarro, Olivier Goury, Gang Zheng, Member, IEEE, Thor Morales Bieze and Christian Duriez, \\ Member, IEEE
}

\begin{abstract}
In this paper, we introduce a new pneumatic mechanosensor dedicated to Soft Robotics and propose a generic method to reconstruct the magnitude of a contact-force acting on it. Changes in cavity volumes inside a soft silicon pad are measured by air-flow sensors. The resulting mechanosensor is characterized by its high sensitivity, repeatability, dynamic range and accurate localization capability in 2D. Using a regression found by machine learning techniques we can predict the contact location and force magnitude accurately when the force magnitudes are within the range of the training data. To be able to provide a more general model, a novel approach based on a Finite Element Method (FEM) is introduced. We formulate an optimization problem, which yields the contact load that best explains the observed changes in cavity volumes. This method makes no assumptions on the force range, the shape of the soft pad or the shape of its cavities. The prediction of the force also results in a model for the deformation of the soft pad. We characterize our sensor and evaluate two designs, a soft pad and a kidney-shaped sensor, in different scenarios.
\end{abstract}

Index Terms-Soft Sensors and Actuators; Force and Tactile Sensing; Modeling, Control, and Learning for Soft Robots

\section{INTRODUCTION}

O NE of the big current challenges in Soft Robotics is the handling of contact situations. On the one hand, researchers are exploring new sensor technologies that are capable of detecting contacts and deformations in compliant structures especially. On the other hand, there is also the need to process the sensor signals to help modeling the robot. There is already a large body of work showing different sensors and measuring principles, but an important part of the modeling of force and deformation is still done using regression techniques rather than generic models.

In recent years it has been shown that it is possible to use the FEM (Finite Element Method) to simulate the behavior of soft structures at interactive update rates, and to transfer these results to real robots made out of deformable materials. In the work of our team, these methods are implemented within

Manuscript received: February, 24, 2019; Revised June, 12, 2019; Accepted July, 8, 2019.

This paper was recommended for publication by Editor Kyu-Jin Cho upon evaluation of the Associate Editor and Reviewers' comments. This work was supported by the Region Hauts-de-France, the project COMOROS (ANR17-ERC2-0029), the European Regional Development Fund (ERDF) and the project Inventor (I-SITE ULNE, le programme d'Investissements d'Avenir, Métropole Europeénne de Lille).

All authors are with team DEFROST at Inria Lille - Nord Europe and CRIStAL - Centre de Recherche en Informatique Signal et Automatique de Lille, France \{stefan.escaida-navarro, olivier.goury, gang.zheng, thor.morales-bieze, christian.duriez\}einria.fr.

Digital Object Identifier (DOI): see top of this page.
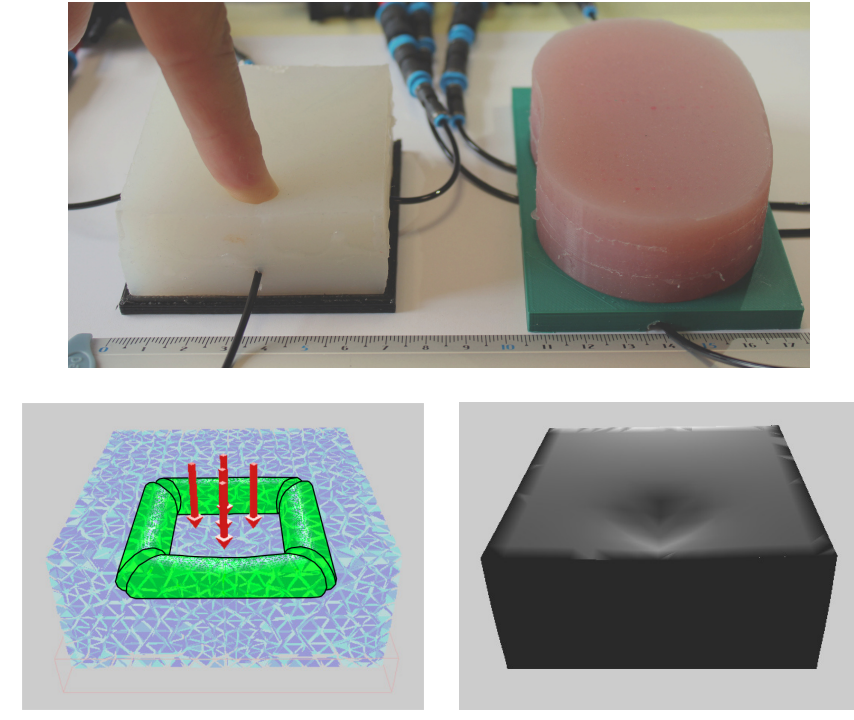

Fig. 1. In this work we show two designs of a novel soft mechanosensor made out of silicone (top, a soft pad and a kidney). When an external force is applied, the volume of cavities embedded in the silicone changes (left). This change in volume is registered through air-flow sensors. Using machine learning and FEM-based techniques, we show that it is possible to estimate the location and magnitude of an external force on the mechanosensor. Using the FEM also yields an estimation of the deformation of the sensor (left and right)

the multiphysics simulation framework SOFA [1], [2]. ${ }^{1}$ In this approach we see an opportunity to advance towards generic models that can integrate measurements from a diversity of sensors. In fact, vision-based approaches, i.e. using extrinsic sensing, have been used in the previous work of our team to estimate interaction forces with soft robot structures [3]. Respective methods for including the contacts in direct and inverse simulation have also been developed [4]. However, until now we have not explored intrinsic sensing modalities.

This work has two main contributions. The first one is to show the design of novel pneumatic mechanosensors, a soft pad and a kidney, pictured in Fig. 1. They are blocks made out of silicone with generic shape and internal cavities that are connected to the outside world through tubes. The sensors work by measuring the changes of cavity volumes with airflow sensors. We show that the sensors have a very good localization capability. For example, we obtain a mean error of $0.79 \mathrm{~mm}$ for the soft pad. With respect to force estimation, we obtain an error of down to $3.52 \%$ over the whole area of

${ }^{1}$ https://www.sofa-framework.org/ 
the soft pad sensor when using an artificial neural neutwork (ANN) to model its behavior. The measurement principle is also responsible for the availability of a high force dynamic range. The second contribution is a novel method for estimating the magnitude of a contact force and the resulting deformation using a FEM-based approach (see also Fig. 1). We model the sensor with the help of the FEM and numerically find the force magnitude that best explains the changes in volume observed. The method is generic, i.e. it makes no assumptions on the geometry of the sensor or the cavities inside it and can be used without relying on a previously found regression for the force. For example, we reach a force reconstruction error of $7.72 \%$ with the soft pad, while having a better generalization than the ANN-based approach. Results for the kidney sensor are similar. The optimization-based approach is a first step towards a fully numerical estimation of all force parameters including the localization. We evaluate our sensor designs in detail.

The remainder of the paper is organized as follows: in the next section we discuss the related work from the field. In Sec. III we describe the design of the sensor and its characterization. In Sec. IV we introduce the approaches for force reconstruction. Then, in Sec. V, we show an evaluation of our soft pad and kidney sensor's performance in different scenarios. Finally, in Sec. VI, we summarize our work, give conclusions and an outlook for future research.

\section{STATE OF THE ART}

Recently, many new soft mechanosensors with a variety of measurement principles have been proposed. A 2018 survey paper by Wang et al. discusses many contributions [5]. As can be recognized from this article, the most popular sensing principles are based on changes in electrical properties of materials to transduce mechanoperception, i. e. tactile information (force, slip) and proprioception (shape, bending). Examples are due to Lucarotti et al. [6], Truby et al. [7] and Wall et al. [8]. Other measuring principles discussed are optical, e.g. [9]. In these works the sensors are modeled with regression techniques. Also, these designs require one or more additional manufacturing steps that bond the sensing elements inside the soft structure by glueing, casting, etc. By comparison, fabricating a soft structure with a cavity for pneumatic sensing is streamlined with the fabrication of the structure itself.

Pneumatic sensing is less prominently featured in the literature, indicating that it is an underexplored option in Soft Robotics. Recent contributions in this field are due to Soter et al., Yang et al., Gong et al. and Choi et al. [10], [11], [12], [13]. Soter et al. propose a design in which cavities inside a soft pad are are filled with a colored liquid [10]. The cavities are connected to an (analog) thermometer-like display for each cavity. Pressing on the cavities makes the level of the liquid in the display rise. This change in level is captured by a camera for all cavities at once and measured as change in pressure/volume. In terms of modeling, the sensor is identified at a single contact location through a linear regression. Yang et al. propose a design of a soft sensor with a cuboid-shaped cavity [11]. With a beam model they show that measuring
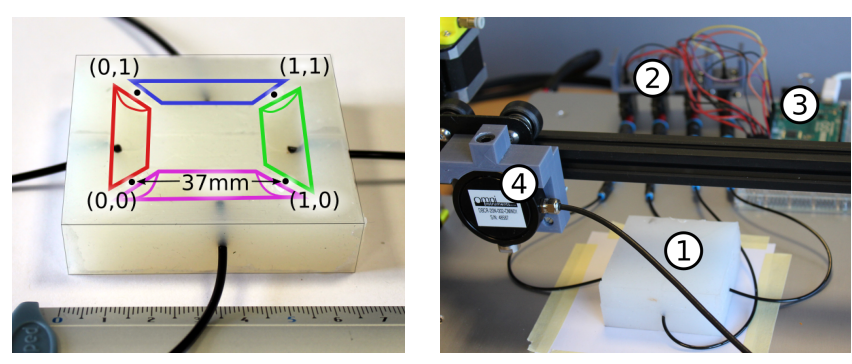

Fig. 2. Left: One half of our sensor, with a demonstration of the dimensions of the soft pad as well as the arrangement and geometry of its cavities. Right: The test bench used to evaluate the performance of the sensor, which is based around a 3D printer and a force sensor: (1) is the soft pad, (2) are the air-flow sensors, (3) is the Arduino board used to digitize the sensor values and (4) is the force sensor.

pressure inside the cavity can be used to detect contact force or curvature of the cavity (not at the same time) with quite accurate results. However, the range of forces tested is rather limited $(20 \mathrm{~g}-130 \mathrm{~g})$. Also, in their work the loading of the sensor is restricted to happen only along one direction, because the model can not account for general deformations of the cavity. A comparable design is due to Gong et al. [12] where a deformable tube (bladder) is used to implement a tactile sensor. The authors identify the pressure vs. force behavior as well as investigating the capabilities of slip detection through FFT-analysis, i.e. retrieving the frequencies of vibrations that are detected by the pressure sensor. Yet, due to the lack of spatial resolution, this sensor can not distinguish the location of the force or slip event. Finally, Choi et al. propose a three-axis force sensor based on pressure sensing inside of three cavities arranged symmetrically in a circle at $120^{\circ}$ [13]. The authors show a method to de-correlate the signals of the cavities in order to find the independent axes and magnitude of a force acting upon the configuration. This is an example of use of a spatial resolution or array to detect forces, but the reconstruction of the forces is restricted to forces applied at the center-point.

All in all, the pneumatic sensors proposed so far in literature lack the use of a spatial resolution to locate the points of contact as well as a seamless integration into a simulation framework for generic sensor modeling, as proposed in this document. The lack of generic modeling is also true for the other measurement principles discussed above. Finally, to the best of our knowledge, no recent works exist that use air-flow measurements as we do in this work, which in our opinion is key to capture the deformation of our soft pad sensor and responsible for a high sensitivity, repeatability and dynamic range.

\section{SEnsor Design AND ChARACTERIZATION}

In this section we provide the design and characterization of our mechanosensor as well as description of the test bench used (see Figs. 1, 2 and 11).

\section{A. Sensor Design}

The design of the soft pad, which is shown in Fig. 2 (left), is relatively straightforward. The figure shows one of 

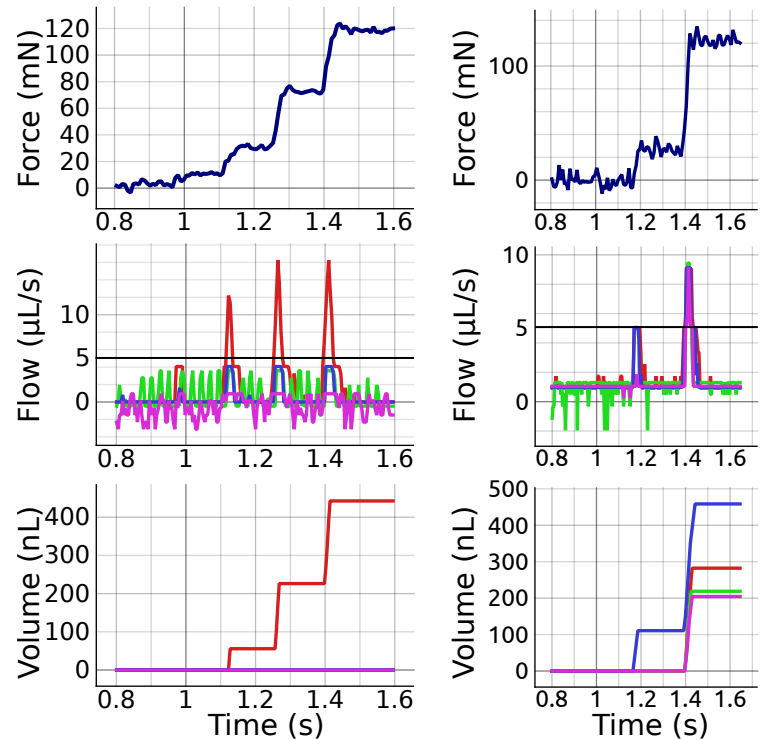

Fig. 3. Left: An iterative sampling to demonstrate the sensitivity at location $(0,0.5)$, directly in the middle over the red cavity. Right: An iterative sampling at the center, the least sensitive part of the sensor, i. e. at coordinates $(0.5,0.5)$. The threshold for contact detection of $5 \mu \mathrm{Ls}^{-1}$ is also shown. Please refer to Fig. 2 for the correspondence of the signal colors to each cavity.

two symmetric parts that are glued together to form the soft pad sensor. The arrangement of the cavities enables the localization of a contact along two directions, i. e. on a plane. The fabrication of the kidney is analogous. We define the sensor origin $(0,0)$ at the border between the left (red) and the bottom (magenta) cavity. The distance between the corner points of this array is $37 \mathrm{~mm}$. We chose elongated, cylindrical cavities over spherical cavities, because the distance of the center point of the pad to any of the cavities is smaller. This allows to maintain the best possible sensitivity at the center point (see also Sec. III-C). The colors shown for the cavities correspond to the colors provided in the signal vs. time plots throughout the paper, see for example Fig. 3.

To measure the changes in volume, we use the air flow sensor D6F-P0001A1 by Omron [14, pp. 25-27]. Its measurement principle is shorty illustrated in the data-sheet [14, $\mathrm{p}$. 3]. For our scenario, measuring air-flow is advantageous in comparison to measuring pressure changes, because we do not have to use the ideal gas law to find the change in volume, we only have to integrate the signal over time. In this way we do not have to know the exact volume of the tubing and can be more resilient to inaccuracies in the fabrication process. The sensors are rated for measuring air (gas) flow between 0 and $0.1 \mathrm{~L} \mathrm{~min}^{-1}\left(=1.66 \mathrm{~mL} \mathrm{~s}^{-1}\right)$. In practice, this allows for a fairly high sensitivity to contact and dynamic range, as discussed in Sec. III-C. The Arduino transmits digitized data at a rate of about 230 FPS with four sensors. Before that, a mean filter with a window size of 5 frames is used to smooth the signal on the microcontroller.

\section{B. Test Bench}

Our test bench consists of a CR10 3D printer by Creality and a Omni-Instruments DBCR force sensor rated for measuring forces between $-20 \mathrm{~N}$ and $20 \mathrm{~N}(< \pm 0.03 \%$ FS repeatability, $< \pm 0.05 \%$ FS non-linearity) [15] that is attached to the carriage of the printer instead of the printing nozzle. The sensor is read out by a PhidgetBride 1046_0B ${ }^{2}$ load cell digitizer. Attached to the force sensor is a load-button with a width of $12.5 \mathrm{~mm}$, which is roughly the size of a index finger tip. The placement of the sensor can be seen in Fig. 2 (right). The positioning accuracy in the $z$-axis of the CR10 is theoretically $0.01 \mathrm{~mm}$ for microsteps of the stepper motor. Full steps achieve a displacement of $0.04 \mathrm{~mm}$. To register our sensor to the printer, we attach a pen to the force sensor. This enables us to trace an accurate outline of the soft pad sensor on a white piece of paper in order to place it at a known location on the test bench.

\section{Sensitivity}

To give an idea about the sensitivity of the air-flow measurements and how they relate to the forces exerted, we present example data in Fig. 3. On the left, the figure shows measurements for the most sensitive spots of the sensor, i.e. directly above any of the cavities. Fig. 3 (left) shows an example for the red cavity. For each force level, the printer is moved by $0.04 \mathrm{~mm}$ downwards. A change in force down to $40 \mathrm{mN}$ can be registered by the flow sensors at this position when using a threshold of $5 \mu \mathrm{Ls}^{-1}$ for contact detection, corresponding to volume changes in the range of nano liters. This is roughly equivalent to $4 \mathrm{~g}$, i. e. very light touch. The threshold for the air-flow signal is used to avoid false readings due to the noise in the signal. On the right, the figure shows the least sensitive spot of the sensor, i.e. directly in the center. Here, the printer is moved by steps of $0.1 \mathrm{~mm}$. A difference of about $100 \mathrm{mN}(\approx 10 \mathrm{~g})$ is necessary to surpass the established flow threshold for all cavities. In the force reading at the $1.2 \mathrm{~s}$ mark, a first touch event is recognizeable. It is barely detected by the blue sensor that triggers. At the $1.4 \mathrm{~s}$ mark, where all sensors trigger, it has therefore already accumulated a small volume reading. Together with the evaluation discussed in Sec. V it becomes clear that the soft pad sensor proposed here is capable of detecting contact situations with a force range of about 2 orders of magnitude (presumably more, but not tested). Finally, it is worth noting that the flow measurements are sensitive to the rate of change of the force, which is something that needs further attention for future works. Another variable that will modulate sensitivity is material stiffness. Softer materials will deform more and therefore increase sensitivity.

\section{Repeatability}

We conducted an experiment with repeated indentation on the soft pad directly above one cavity with three indentation levels. For each level the soft pad was sampled 10 times. The statistics of the experiment are summarized in Table I. The repeatability of the soft pad sensor is high, i.e. better than $0.5 \%$ standard deviation for the volume estimation. This number is consistent with the data sheet of the air-flow sensor, where it is indicated that the repeatability is within $\pm 1.0 \% \mathrm{FS}$

\footnotetext{
${ }^{2} \mathrm{https}: / /$ www.phidgets.com/?tier=3\&catid=98\&pcid=78\&prodid $=1027$
} 


\begin{tabular}{|c|c|c|c|c|c|c|}
\hline & \multicolumn{3}{|c|}{ Force $(\mathrm{N})$} & \multicolumn{3}{c|}{ Volume $(\mu \mathrm{L})$} \\
\hline Level & mean & std & $\%$ & mean & std & $\%$ \\
\hline 1 & 3.30 & 0.01 & 0.26 & 37.37 & 0.11 & 0.30 \\
\hline 2 & 6.19 & 0.02 & 0.35 & 71.50 & 0.30 & 0.42 \\
\hline 3 & 9.42 & 0.04 & 0.44 & 114.54 & 0.54 & 0.47 \\
\hline
\end{tabular}

REPEATABILITY TEST FOR THREE DIFFERENT INDENTATION LEVELS

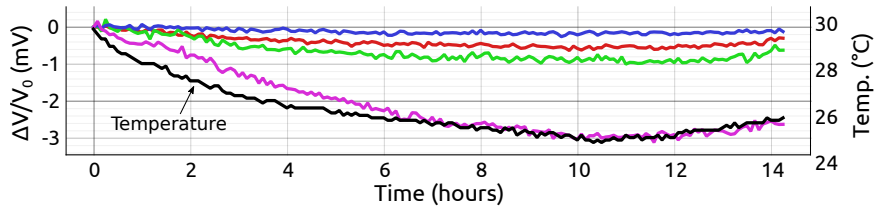

Fig. 4. A plot showing the offset-voltage drift related to time and temperature for four air-flow sensors over the course of 14 hours.

deviation $[14$, p. 26]. We think this is the factor that allows precise localization with our sensors, as seen in the evaluation (see Sec. V).

\section{E. Drift Related to Time and Temperature}

According to the data sheet of the air-flow sensors, a $\pm 5 \%$ FS drift can be expected in the range of $-10^{\circ} \mathrm{C}$ and $60^{\circ} \mathrm{C}[14$, p. 26]. A time related drift is not mentioned in the document. We let four sensors run without any contact interactions for 14 continuous hours in which the temperature reached a maximum of about $5^{\circ} \mathrm{C}$ difference. As can be seen in Fig. 4 there is a very small drift of only a few $\mathrm{mV}$ in the offset voltage, which is correlated to the temperature change. It is more significant for one of the sensors than for the other three, indicating fabrication tolerances. The maximum drift of $3 \mathrm{mV}$ translates to a drift in air-flow of $2.5 \mu \mathrm{L} \mathrm{s}^{-1}$, which is lower than the threshold established for contact, i.e. $5 \mu \mathrm{Ls}^{-1}$ (see Fig. 3). We think that it is unproblematic to adjust these offsets regularly to account for the small changes related to time and temperature drift. Silicone changes its mechanical properties only beyond $100^{\circ} \mathrm{C}$, so it is not a factor of immediate concern.

\section{F. Hysteresis}

Measuring the volume through the air-flow sensing principle is highly repeatable as shown in Sec. III-D. However, it is
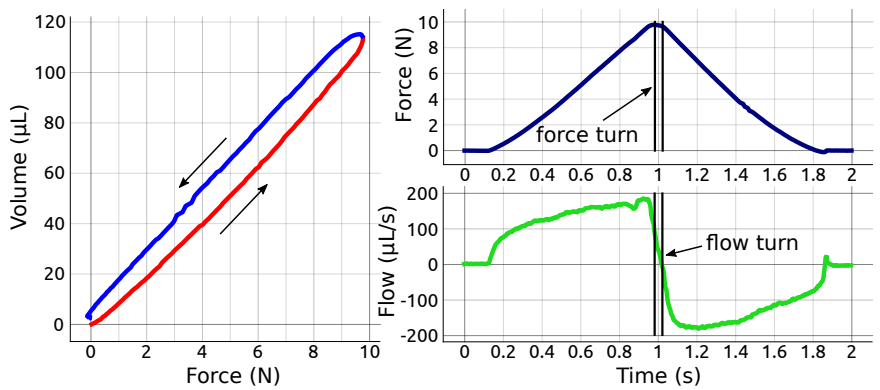

Fig. 5. Left: Hysteresis curve for a load of about 10N. Right: Signals for force and air-flow illustrating the different moments in time at which the unloading is first detected in the force and in the air-flow.
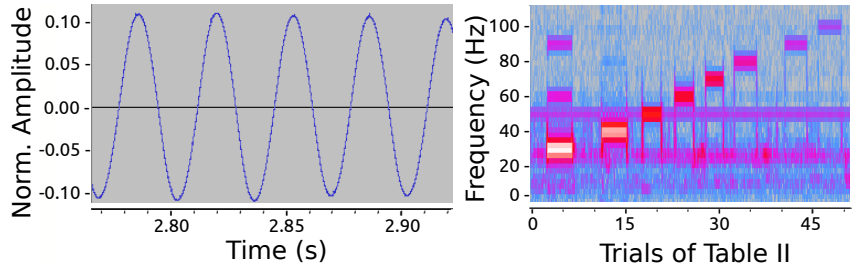

Fig. 6. Left: The wave signal of the pad being vibrated at a frequency of $30 \mathrm{~Hz}$ as captured by a soundcard. Right: Spectrogram of the soft pad being exposed to different vibration frequencies (see Table II).

\begin{tabular}{|c|c|c|c|c|c|c|c|c|}
\hline F (Hz) & 30 & 40 & 50 & 60 & 70 & 80 & 90 & 100 \\
\hline A (dB) & -13 & -18 & -31 & -36 & -38 & -47 & -55 & -60 \\
\hline
\end{tabular}

AIR-FLOW SIGNAL RESPONSES FOR VIBRATION AT CONSTANT AMPLITUDE

prone to hysteresis behavior as shown by Fig. 5. There are at least two possible explanations for this behavior: First, the accuracy of the air-flow sensor is not as high as its repeatability, i.e. only $\pm 5.0 \%$ FS $[14$, p. 26]. This means the non-linearity in the flow measurement will lead to errors in the integration. For example, the range in which the sensor represents positive flow is likely to behave somewhat differently than the range for negative flow. Second, as the airflow sensor relies on a differential temperature measurement it is susceptible to inertia effects. As shown on Fig. 5 (right), after the moment when the unloading phase starts according to the force readings (force turn, force starts decreasing), the flow readings continue to be positive for a brief moment $(0.05 \mathrm{~s})$, i. e. contributing to measure increasing volume. This effectively generates an offset for the unloading curve with respect to the loading curve. In addition, the value for the volume does not go entirely back to 0 when there is no more load. In many practical use cases, however, it is possible to detect a full release due to the high negative air flow. To provide an absolute reference in all scenarios it would be necessary to complement the air-flow measurements with an absolute measurement principle like pressure sensing.

\section{G. Bandwidth}

To obtain an idea of the bandwidth of vibration frequencies that can be detected by soft pad, we conducted an experiment exciting the soft pad with vibrations at different frequencies ranging from $30 \mathrm{~Hz}$ to $100 \mathrm{~Hz}$. The soft pad was placed on top of a studio speaker with linear reproduction characteristic between $30 \mathrm{~Hz}$ and $22 \mathrm{kHz}$. An additional weight was placed on top of the soft pad to improve the transmission of the vibration from the chassis of the speakers to the soft pad. The analog voltage output from one air-flow sensor was connected through a jack plug to the input of a sound card sampling at $48 \mathrm{kHz}$. Fig. 6 shows an example of the wave recorded for the vibration at $30 \mathrm{~Hz}$ as well as a spectrogram for all the frequencies testet. The vibration amplitudes obtained for each frequency are listed in Table II.

From the results it is recognizeable that the soft pad together with the air-flow sensor act as a low-pass filter with significant dampening, i.e. $47 \mathrm{~dB}$ from $30 \mathrm{~Hz}$ to $100 \mathrm{~Hz}$. However, vibrations with frequencies as high as $100 \mathrm{~Hz}$ can still be clearly 
found by a spectral analysis, showing the potential for slip detection for example.

\section{Sensor Modeling Techniques}

In this section we describe the methods used to predict the force parameters from the measured volumes using an Artificial Neural Network-based approach and a FEM-based approach.

\section{A. ANN-based Approach for Force Localization and Magni- tude Estimation Using Volume Measurements}

Given there is a (single) contact with the sensor, the goal of this method is to predict the contact's location and the magnitude of the force applied from the changes in volume observed. In our case a simple Artificial Neural Network (ANN) approach is sufficient, as the input and output dimensionalities are low (four or six input parameters, three outputs). The training data is used to train a feed-forward ANN implemented in Keras with a Tensorflow back-end. ${ }^{3}$ The four or six volumes of each cavity are connected to a layer of 128 neurons. These are in turn connected to a hidden layer of 40 neurons, which are finally connected to the output layer that predicts $x$ and $y$ coordinates and a corresponding force. The ANN is trained with the goal of minimizing the squared error of the outputs against ground truth data in a given data set. The training is done using gradient back propagation. This method by itself, i. e. without the use of an additional linear elastic model, does not predict the deformation of the soft sensor.

\section{B. Numerical Approach for Force Magnitude Estimation Us- ing Volume Measurements}

The goal of this approach is to offer an alternative to the force magnitude estimation based on regression techniques with the advantage of better generalization. The idea is to use a numerical model of the physics (FEM) and an optimization method to find the force magnitude that best explains the volume changes. It is this approach that will simultaneously yield a model for the deformation of the soft sensor. At the moment this method cannot predict the contact position from the volumes, which is assumed to be a given. It is, however, a first step towards a fully numerical-based force parameter estimation.

1) Online FEM: We use the FEM, which yields the forces $\mathbb{F}(q)$, given that the nodes of the FEM mesh are at positions $q$ (see Fig. 1 left). The model used for the silicone is linear elastic with a corotational formulation (efficient, physically plausible finite elements [16]) that can be evaluated at interactive or near interactive update rates, depending mostly on the number of elements. The model is characterized by Young's Modulus and Poisson's Ratio. During each step $i$ of the simulation, a linearization of the internal forces is computed:

$$
\mathbb{F}\left(q_{i}\right) \approx \mathbb{F}\left(q_{i-1}\right)+K\left(q_{i-1}\right) d q
$$

where $d q=q_{i}-q_{i-1}$ is the displacement of the nodes and $K=\frac{\partial \mathbb{F}\left(q_{i-1}\right)}{\partial q}$ is the tangential stiffness matrix for the current

\footnotetext{
${ }^{3}$ https://keras.io/
}

node positions $q$. To complete the picture, external forces are included:

$$
-K\left(q_{i-1}\right) d q=\mathbb{P}+\mathbb{F}\left(q_{i-1}\right)+H^{T} \lambda_{f} .
$$

$H_{f}^{T} \lambda_{f}$ is the expression that gathers constraining forces, such as contacts or external controlled inputs, i.e. actuators. $\mathbb{P}$ represents known external forces, such as gravity. Then, (2) is solved under the assumption of static equilibrium, delivering a motion that is a succession of quasi static states. In this framework, we can in principle map the quantities measured by the pneumatic sensors to force constraints in $H_{f}^{T} \lambda_{f}$ if we can derive an estimate of the external force location and magnitude from the sensor values. Please refer to [1] for a more in-depth discussion about the FEM formulation used here.

2) Finding the Contact Force Magnitude through Optimization: Using inverse problem solving, we can optimize for the force magnitude that best explains the volume change given that the contact is at a specific location. In other words, the contact can be seen as a new force constraint for which the magnitude should be determined. The estimated deformation of the soft pad sensor is the one that includes this force condition.

Let $\Phi_{i}(q)$ be the function that maps the positions of the nodes to the volume of a cavity with index $i$. $\Phi_{i}(q)$ can be calculated using the triangles of the mesh defining the cavity and is already implemented in SOFA. To find the relation between the change in an external force $\Delta \lambda_{f}$ applied to the object and the change in the (simulated) volume $\Delta V_{\text {sim }}$ we can write:

$$
\Delta V_{\text {sim }}=\frac{\partial \Phi_{i}(q)}{\partial q} K^{-1} H^{T} \Delta \lambda_{f} .
$$

That is to say that first the changes of forces are mapped to the corresponding nodes through $H^{T}$. The tangential compliance matrix $K^{-1}$ transforms these forces to displacements, which finally can be mapped to changes in volume through the derivative of $\Phi$ with respect to $q$. For $\frac{\partial \Phi_{i}(q)}{\partial q} K^{-1} H^{T}$ we write $W_{i, v f}$, which is the matrix that directly maps changes in force to changes in volume. Now, we can formulate the optimization problem. We want to find the actuation force magnitude $\Delta \lambda_{f}$ that best explains the sum of changes in the simulated volumes $\Delta V_{i, s i m}=W_{i, v f} \Delta \lambda_{f}$ so that the difference to the sum of the real changes in volume $\Delta V_{i, \text { real }}$ is minimized:

$$
\Delta \lambda_{\text {out }}=\underset{\Delta \lambda_{f}}{\arg \min }\left\|\sum W_{i, v f} \Delta \lambda_{f}-\sum \Delta V_{i, \text { real }}\right\|^{2} .
$$

Finally, we can write out the squared expression. There, we can ignore the constant term corresponding to the sum of the changes in real volumes for the optimization. We also substitute $\sum W_{i, v f}$ for $S$ and $\sum \Delta V_{i, \text { real }}$ for $\Delta V_{\text {total }}$ for notation convenience in (4), so we arrive at:

$$
\Delta \lambda_{\text {out }}=\underset{\Delta \lambda_{f}}{\arg \min }\left(\Delta \lambda_{f}^{T} S^{T} S \Delta \lambda_{f}-2 \Delta \lambda_{f}^{T} S^{T} \Delta V_{\text {total }}\right) .
$$

This quadratic program (QP) can be solved using a QP-solver with the method presented in [2]. ${ }^{4}$

\footnotetext{
${ }^{4}$ In this work $\Delta \lambda_{f}$ in (5) is actually a scalar, but we provided the formulation for the general case where $\Delta \lambda_{f}$ is a vector.
} 

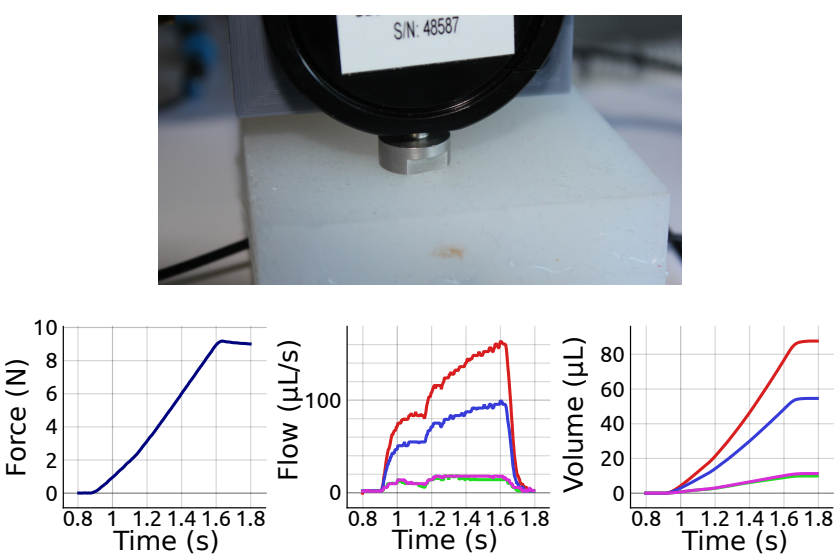

Fig. 7. Top: A close-up of the sampling procedure that is used to collect training and test data for our evaluation scenarios. Bottom: An example for a time-series corresponding to one of the points in the training set. The coordinates of the point are $(0,0.8)$ on the soft pad.

\section{Evaluation}

In this section, we discuss the performance in localization and force estimation using the soft pad and the kidney sensor. We show in three scenarios how force can be estimated from the changes in volumes of the cavities. Fig. 7 shows a closeup of the sampling procedure using the setup described in Sec. III-B and an example for data collected for training purposes.

\section{A. Experiment Description}

In this section we present the results of the force reconstruction for our sensors. There are three scenarios in which the soft pad and the kidney sensor were tested:

- R(eal)-ANN: An ANN-model is trained on real data (see Sec. IV-A) collected with the setup described in Sec. III-B. The model predicts both position and force when provided with changes in volumes as inputs.

- Opti(mization): The same model as in the previous scenario is used to predict only the contact location. The force magnitude is reconstructed using the optimization method presented in Sec. IV-B.

- Transfer: An ANN-model is trained on simulated data, that is, the sampling procedure is simulated in SOFA. The force position is predicted by the simulation-based ANN and the magnitude is reconstructed, again, using the method described in Sec. IV-B. This is a transfer learning approach.

To train the ANN models, data is collected with the real setup or in simulation by sampling the sensor in regular intervals, with 2 or 3 indentation depths per point. First, the sensors are sampled in $3.7 \mathrm{~mm}$ intervals $($ a $11 \times 11$ grid on the soft pad) with three force levels: approx. $0.5 \mathrm{~N}, 3 \mathrm{~N}$ and $9 \mathrm{~N}$ and then with an interval of $6.1 \mathrm{~mm}$ (a $7 \times 7$ grid) with approx. $4 \mathrm{~N}$ and $10 \mathrm{~N}$. The two or three levels correspond to two or three target indentations, i. e. all the samples of one level have the same indentation depth, but have varying forces depending on where on the array the sample is taken, which is mainly due to the cavities locally affecting the stiffness.

\begin{tabular}{|c|c|c|c|c|c|}
\hline & \multicolumn{2}{|c|}{ Pos. err. (mm) } & & \multicolumn{2}{|c|}{ F err. (N) } \\
Scenario & mean & std & F err. $(\%)$ & mean & std \\
\hline \hline$R-A N N_{s p}(A l l)$ & 0.79 & 0.74 & 11.46 & 1.46 & 1.85 \\
\hline$R-A N N_{s p}<11 N$ & 0.62 & 0.60 & 3.52 & 0.21 & 0.20 \\
\hline Opti $_{s p}($ All $)$ & 0.79 & 0.74 & 7.72 & 0.68 & 0.66 \\
\hline Opti $_{s p}>11 N$ & 1.12 & 0.86 & 7.95 & 1.15 & 0.82 \\
\hline Transfer $_{s p}($ All $)$ & 2.88 & 2.03 & 8.34 & 0.74 & 0.74 \\
\hline
\end{tabular}

TABLE III

MEAN LOCALIZATION AND FORCE RECONSTRUCTION ERRORS FOR THE DIFFERENT SCENARIOS EVALUATED

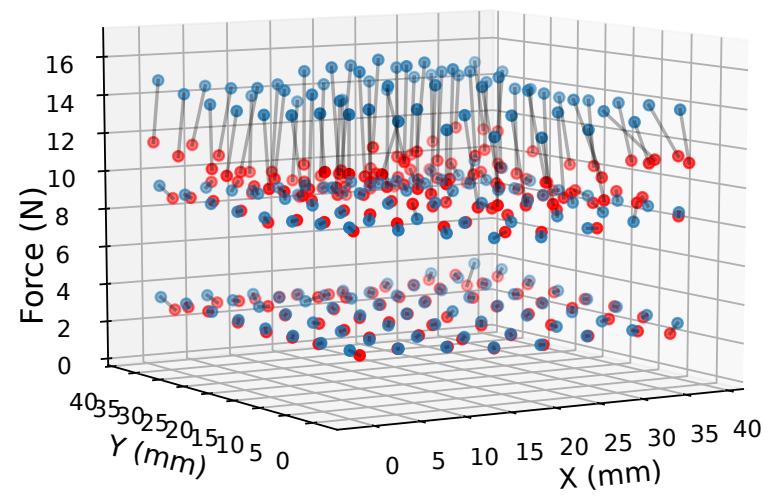

Fig. 8. Points of the test set (blue) plotted against the predicted output (red) in the $R-A N N_{s p}$ scenario. Transparent lines connect corresponding points from the test set to their predicted values.

Each of the three scenarios is evaluated with a data-set that is aimed at testing the performance and generalization capabilities of each approach. The test set consists of data collected by sampling in intervals of $5.3 \mathrm{~mm}$ (a $8 \times 8$ grid) with three force levels: approx. $3 \mathrm{~N}, 10 \mathrm{~N}$ and $15 \mathrm{~N}$. Therefore, this set is mostly based on different positions and indentation/force levels than the data used for training. Also, while the training data contains force levels between approx. $0.5 \mathrm{~N}$ and $10 \mathrm{~N}$, the test set goes beyond that range. This will allow us to see how the different approaches generalize in terms of localization and force magnitude.

\section{B. Results Soft Pad}

The results of the different approaches for the soft pad are summarized by Table III. Figs. 8-10 show the detail of each

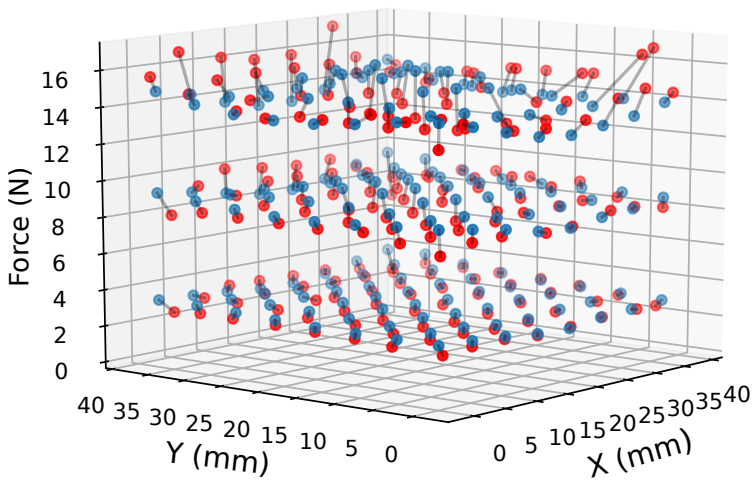

Fig. 9. Same as Fig. 8 for the $O p t i_{s p}$ scenario. 


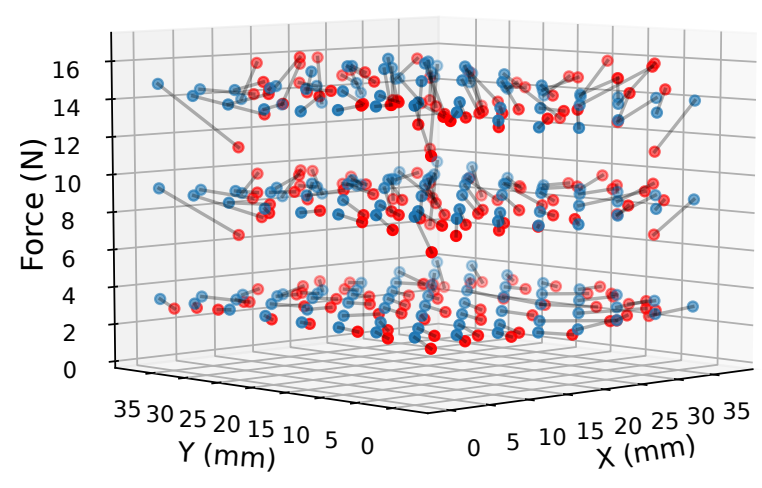

Fig. 10. Same as Fig. 8 for the Transfer ${ }_{s p}$ scenario.

test sample compared to its predicted value. First, from the results it becomes clear that the localization accuracy possible with the soft pad sensor is high, that is, only a $0.79 \mathrm{~mm}$ error for all the data in the $R-A N N_{s p}$ scenario. We attribute this to the sensitivity and repeatability provided by the air-flow sensors, meaning the volume measurements serve to predict a contact's exact position.

With respect to the force reconstruction, the $R-A N N_{s p}<11 N$ scenario shows the best performance in correctly predicting the test data (3.52\% mean error). Here, the forces are within the margin established for the training data. It is also the best predictor for the position of the contact, with a mean error of only $0.62 \mathrm{~mm}$. It is worth noting that tuning the parameters of the ANN did not significantly affect the results. We deal with low-dimensionality inputs and outputs, highly repeatable measurements (see Sec. III-D) as well as a good amount of training data. These are very promising conditions for any statistical learning approach. Therefore, we consider that these force magnitude and localization results are a reflection of actual behavior of the soft pad sensor, being a baseline for the possible performance. As expected, the network fails at predicting forces that are significantly outside of the force range for the training data, as can be seen by Fig. 9. Considering all test data $\left(R-A N N_{s p}\right)$, the deviation of the absolute force magnitude is worse than the $O p t i_{s p}$ and Transfer $_{s p}$ scenarios $(1.46 \mathrm{~N}$ against $0.68 \mathrm{~N}$ and $0.74 \mathrm{~N})$.

The $O p t i_{s p}$ scenario shows the most consistent performance overall, as can be seen in Fig. 9. For the prediction of the position it relies on the previous approach, so the results are the same in that category. While it is outperformed by the $R$ $A N N_{s p}$ scenario for data points lying within the force intervals considered for training, it generalizes better to forces that had previously not been seen. In fact, when isolating these points in the scenario $O p t i_{s p}>11 N$, we see that in terms of percentual error the values are not significantly worse $(7.72 \%$ vs. $7.95 \%)$, indicating a strong capability of generalization. As a result, it outperforms the $R-A N N_{s p}$ scenario in terms of percentual force error. We should also mention that these results show that the ANN-based approach generalizes well in terms of localization accuracy, with a mean error of $1.12 \mathrm{~mm}$ (see $O p t i_{s p}>11 N$ ). This is plausible as the localization depends mostly on the relative magnitudes of the volumes, not on the total amplitude.

Finally, the Transfer $_{s p}$ scenario delivers acceptable results

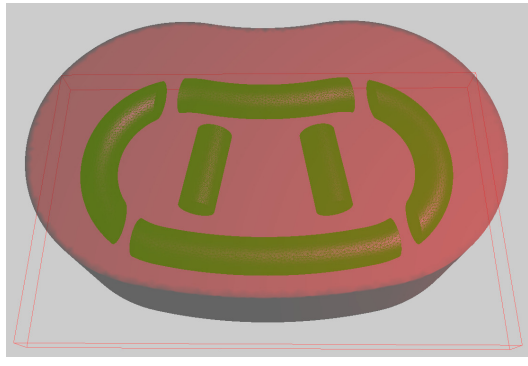

Fig. 11. The kidney sensor and the layout of its cavities.

at the current state, but there is a significant deterioration regarding the localization compared to $R-A N N_{s p}$. However, with an error of $2.88 \mathrm{~mm}$ it is still usable. With the less accurate localization, the force magnitude estimation also deteriorates $(8.43 \%$ mean error), while still being within reasonable margins and generalizing well to unforeseen forces (better than $R-A N N_{s p}$ ). This lets us conclude that for the force estimation the most important factor is the total amount of volume change (deformation), rather than where exactly the contact happens.

\section{Results Kidney}

The kidney-shaped sensor is motivated by the idea of creating active phantoms with sensing capabilities or similar devices for the purpose of medical training. The kidney differs from the soft pad in its shape but also in the amount of cavities embedded. As seen in Fig. 11 there are six cavities arranged to follow the shape of the sensor and to cover the area where touch interactions are expected. Beyond that, the cavities are generally somewhat closer to each other than in the case of the soft pad. In order to guarantee a good sampling rate of the air-flow sensors, two Arduinos where used.

The results for the kidney are shown in Table IV. In the greater picture these results are very similar to the ones obtained with the soft pad. The $R-A N N_{k}$ is the best in the interpolation regime, but is outperformed by the other approaches when the task is to generalize to unforseen forces. Overall the accuracy of contact location is slightly better than for the soft pad. For example, the mean error for $R-A N N_{k}<$ $11 N$ is only $0.56 \mathrm{~mm}$ while it was $0.62 \mathrm{~mm}$ for $R-A N N_{s p}$. A plot showing the data for $R-A N N_{k}<11 N$ is shown in Fig. 12 . To us, this is an effect of the cavities being closer together.

It is interesting that the result for the localization error for Transfer $_{k}$ is noticeably better than for the soft pad (a mean error of $1.94 \mathrm{~mm}$ vs $2.88 \mathrm{~mm}$ ). Again, the fact that cavities are closer together and that they are less regular seems to help to inform the machine learning approach, even if it is executed on simulated data. Interestingly, the force prediction is slightly better in the transfer learning-based approach (Transfer ${ }_{k}$ ) than in the optimization-based approach. As seen in Figs. 9 and 10 the force reconstruction tends to deviate most directly above the cavities. Due to the errors in position estimation in Transfer $_{k}$, the location is shifted towards a place were the force estimated is slightly more reliable. However, besides that, the force reconstruction is slightly worse for some 


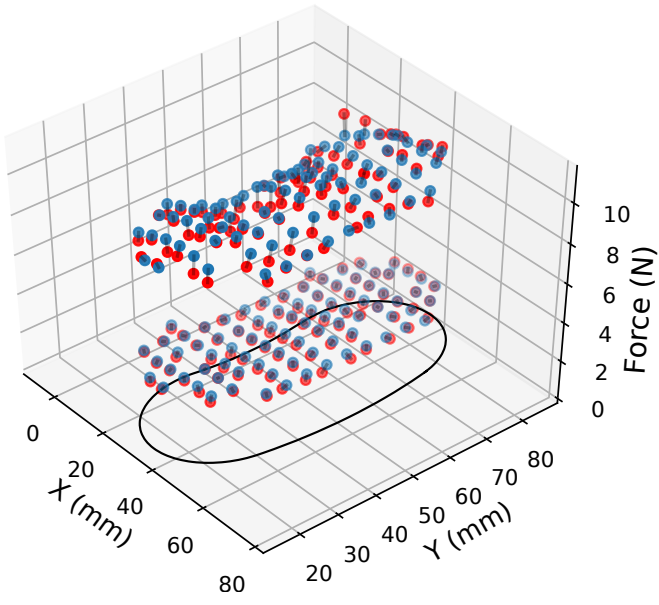

Fig. 12. Same as Fig. 8 for the $R-A N N_{k}<11 N$ scenario. The contour defined by the center of the cavities is shown below the data.

\begin{tabular}{|c|c|c|c|c|c|}
\hline & \multicolumn{2}{|c|}{ Pos. err. (mm) } & & \multicolumn{2}{|c|}{ F err. (N) } \\
Scenario & mean & std & F err. (\%) & mean & std \\
\hline \hline$R$-ANN $N_{k}($ All $)$ & 0.64 & 0.45 & 10.27 & 1.20 & 1.42 \\
\hline$R-A N N_{k}<11 N$ & 0.56 & 0.39 & 4.00 & 0.24 & 0.23 \\
\hline Opti $_{k}($ All $)$ & 0.64 & 0.45 & 7.66 & 0.72 & 0.77 \\
\hline Opti $_{k}>11 N$ & 0.80 & 0.53 & 10.19 & 1.38 & 0.88 \\
\hline Transfer $_{k}($ All $)$ & 1.94 & 0.94 & 6.98 & 0.64 & 0.65 \\
\hline
\end{tabular}

TABLE IV

MEAN LOCALIZATION AND FORCE RECONSTRUCTION ERRORS FOR THE DIFFERENT SCENARIOS EVALUATED KIDNEY

scenarios than in the case of the soft pad. This could be related to the increased variations in the stiffnes of the kidney due to the increased number of cavities.

\section{CONCLUSIONS}

This work has two main contributions: first, we showed novel designs for soft sensors made out of silicone, a soft pad and a kidney shaped sensor. When there is a contact, the volumes of cavities inside the silicone structure change. These changes are measured through air-flow sensors. The air-flow sensors and the cavity arrangement allow for the detection of contact events within a large range of forces and provide precise cues for the force localization, as they provide measurements even for tiny deformations. Second, we compared two approaches for reconstructing the actual magnitude of the force. In the first approach a calibration of the sensors was obtained by training an artificial neural network (ANN) with samples from the real world. In the second approach we used an inverse problem solving approach: with the help of a FEM-model of the sensors we find the force magnitude that best explains the observed change in volume of the cavities. We found that the accuracy of the ANNmodel is best for forces within the range of the samples used for training, but that it does generalize poorly outside of that range. The FEM-based optimization approach is more coarse, but generalizes well to different force magnitudes. In the process, the deformation of the sensor is also estimated. To us, these are encouraging results that could be a contribution to observability and controllability in Soft Robotics.
One of the next steps in our work will be including the force position as a parameter in our optimization framework. This could make our method less reliant on real world data for accurate performance. One of the next steps will also be to apply the approach presented here to a soft robot that is actuated by cables. There we can study the possibility of estimating the contact force location and magnitude and how this force affects the shape of the robot. Another domain of interest is medicine technologies. Our efforts could be used to create smart phantoms of organs that can help assessing the performance of trainees who use them in palpation scenarios. In general, there is still room for improvement of the sensor design in terms of cavity shape and dimensions for the different use cases. Also, as the FEM-techniques progress, the precision in force reconstruction will also improve.

\section{REFERENCES}

[1] C. Duriez, "Control of elastic soft robots based on real-time finite element method," in Robotics and Automation (ICRA), 2013 IEEE International Conference on. IEEE, 2013, pp. 3982-3987.

[2] C. Duriez, E. Coevoet, F. Largilliere, T. Morales-Bieze, Z. Zhang, M. Sanz-Lopez, B. Carrez, D. Marchal, O. Goury, and J. Dequidt, "Framework for online simulation of soft robots with optimizationbased inverse model," in Simulation, Modeling, and Programming for Autonomous Robots (SIMPAR), IEEE International Conference on. IEEE, 2016, pp. 111-118.

[3] Z. Zhang, J. Dequidt, and C. Duriez, "Vision-based sensing of external forces acting on soft robots using finite element method," IEEE Robotics and Automation Letters, vol. 3, no. 3, pp. 1529-1536, 2018.

[4] E. Coevoet, A. Escande, and C. Duriez, "Optimization-based inverse model of soft robots with contact handling," IEEE Robotics and Automation Letters, vol. 2, no. 3, pp. 1413-1419, 2017.

[5] H. Wang, M. Totaro, and L. Beccai, "Toward perceptive soft robots: Progress and challenges," Advanced Science, p. 1800541, 2018.

[6] C. Lucarotti, M. Totaro, A. Sadeghi, B. Mazzolai, and L. Beccai, "Revealing bending and force in a soft body through a plant root inspired approach," Scientific reports, vol. 5, p. 8788, 2015.

[7] R. L. Truby, M. Wehner, A. K. Grosskopf, D. M. Vogt, S. G. Uzel, R. J. Wood, and J. A. Lewis, "Soft somatosensitive actuators via embedded 3d printing," Advanced Materials, vol. 30, no. 15, p. 1706383, 2018.

[8] V. Wall, G. Zöller, and O. Brock, "A method for sensorizing soft actuators and its application to the rbo hand 2," in Robotics and Automation (ICRA), 2017 IEEE International Conference on. IEEE, 2017, pp. 4965-4970.

[9] H. Zhao, K. OBrien, S. Li, and R. F. Shepherd, "Optoelectronically innervated soft prosthetic hand via stretchable optical waveguides," Science Robotics, vol. 1, no. 1, p. eaai7529, 2016.

[10] G. Soter, M. Garrad, A. T. Conn, H. Hauser, and J. Rossiter, "Skinflow: A soft robotic skin based on fluidic transmission," in 2019 2nd IEEE International Conference on Soft Robotics (RoboSoft). IEEE, 2019, pp. 355-360.

[11] H. Yang, Y. Chen, Y. Sun, and L. Hao, "A novel pneumatic soft sensor for measuring contact force and curvature of a soft gripper," Sensors and Actuators A: Physical, vol. 266, pp. 318-327, 2017.

[12] D. Gong, R. He, J. Yu, and G. Zuo, "A pneumatic tactile sensor for co-operative robots," Sensors, vol. 17, no. 11, p. 2592, 2017.

[13] H. Choi, P.-G. Jung, K. Jung, and K. Kong, "Design and fabrication of a soft three-axis force sensor based on radially symmetric pneumatic chambers," in Robotics and Automation (ICRA), 2017 IEEE International Conference on. IEEE, 2017, pp. 5519-5524.

[14] D6F Series, OMRON Corporation. [Online]. Available: https://omronfs. omron.com/en_US/ecb/products/pdf/en-d6f_series.pdf

[15] DBCR Miniature S-Beam Load Cell, APPLIED MEASUREMENTS LTD. [Online]. Available: https://appmeas.co.uk/pdf/load-cells/DBCR. pdf

[16] M. Nesme, P. G. Kry, L. Jeřábková, and F. Faure, "Preserving topology and elasticity for embedded deformable models," in ACM Transactions on Graphics (TOG), vol. 28, no. 3. ACM, 2009, p. 52. 